\title{
Metabolic Changes after Radioiodine Correction of Grade 1 and Grade 2 Subclinical Hyperthyroidism
}

\author{
Lucia Russo $^{a}$ b Thi Ngoc Huyen Nguyen ${ }^{b}$ Aglaia Kyrillib Martin Robin ${ }^{c}$ \\ Pierre Bel Lassen $^{d}$ Rodrigo Moreno-Reyes ${ }^{e}$ Bernard Corvilain ${ }^{b}$ \\ ${ }^{a}$ Department of Medicine, DIMED, Internal Medicine 3, University of Padua, Padova, Italy; ${ }^{\text {DDepartment of }}$ \\ Endocrinology, Erasme University Hospital, Université Libre de Bruxelles, Brussels, Belgium; 'Department of Nuclear \\ Medicine, Erasme University Hospital, Université Libre de Bruxelles, Brussels, Belgium; dDepartment of Urology, \\ Hôpital Universitaire des Enfants Reine Fabiola, Université Libre de Bruxelles, Brussels, Belgium; 'UMRS 1166 \\ (Inserm), Paris, France
}

\section{Keywords}

Multinodular goiter $\cdot$ Hyperthyroidism $\cdot$ Lipids $\cdot$ BMI

\begin{abstract}
Objective: Endogenous subclinical hyperthyroidism (eSCH) is defined by subnormal serum thyroid-stimulating hormone (TSH) level. There is limited evidence of metabolic changes induced by eSCH. The aim of our work was to evaluate changes in $\mathrm{BMI}$ and lipid parameters after radioiodine treatment in patients with grade 1 (TSH: $0.1-0.39 \mathrm{mlU} / \mathrm{L}$ ) and 2 (TSH $<0.1 \mathrm{mlU} / \mathrm{L}$ ) eSCH. Design: A retrospective study was performed on 74 patients with eSCH caused by benign autonomous nodular goiter which was treated with radioiodine. Methods: We assessed BMI, lipids parameters, and TSH after radioiodine therapy. The 12-month follow-up time point was used to compare the primary outcome variables. TSH was measured by the electrochemiluminescence method. Results: After radioiodine therapy, the absolute and relative increases in $\mathrm{BMl}$ at 12 months were significantly higher in the grade 2 group than in the grade 1 group (1.07 \pm 0.27 $\mathrm{kg} / \mathrm{m}^{2}$ vs. $0.26 \pm 0.15 \mathrm{~kg} / \mathrm{m}^{2}$, respectively; $p=0.023$ and 4.01 $\pm 0.98 \%$ vs. $1.01 \pm 0.56 \%$, respectively; $p=0.026$ ). Compared to baseline, significant increases in the levels of total choles-
\end{abstract}

terol and LDL were observed after treatment in the grade 2 eSCH group $(16.7 \pm 4.5 \mathrm{mg} / \mathrm{dL} p<0.01$ and $14.3 \pm 4.1 \mathrm{mg} / \mathrm{dL}$ $p<0.01$, respectively) but not in the grade 1 group. In a multivariate model, a negative correlation was observed between pretreatment TSH levels and absolute BMI gain $(p<$ $0.01)$. Conclusions: After correction of eSCH, increases in BMI and LDL levels were observed only in patients with grade 2 eSCH. Pretreatment serum TSH was the main independent factor associated with BMI changes after radioiodine treatment.

(C) 2021 European Thyroid Association Published by S. Karger AG, Basel

\section{Introduction}

Endogenous subclinical hyperthyroidism $(\mathrm{eSCH})$ is a common disease defined as persistent low serum thyroidstimulating hormone (TSH), with normal levels of free thyroxine (fT4) and tri-iodothyronine (fT3) regardless of the presence or absence of clinical signs of hyperthyroidism [1-4]. Depending on its biological severity, eSCH can be divided into grade $1 \mathrm{eSCH}$, in which the TSH level is mildly suppressed $(0.1 \leq \mathrm{TSH} \leq 0.39 \mathrm{mU} / \mathrm{L})$, and grade 2 $\mathrm{SCH}$, in which the TSH level is $<0.1 \mathrm{mU} / \mathrm{L}[5,6]$. In the 
absence of major intervention trials, most of the reported adverse outcomes of SCH were derived from observational studies and include cardiovascular disease $[7,8]$, decreased bone mineral density [9], and dementia [10].

It is well known that thyroid hormones are important determinants of energy expenditure and appetite regulation and correction of overt clinical hyperthyroidism leads to weight gain [11-15]. However, there is only limited evidence for changes in weight regulation or metabolism induced by eSCH, a state where thyroid hormone levels are normal. Knudsen et al. [16], in one of the few cross-sectional studies to include patients with subclinical thyroid disorders, showed that differences in thyroid function (positive associations found for TSH and fT 4 but not fT3), within what is considered the normal range, are associated with changes in BMI.

However, the impact of SCH correction on metabolic outcomes remains unclear due to the very small number of controlled interventional studies involving only a few subjects [17-19]. Bel Lassen et al. [20], in the largest retrospective study ( $N=225$ patients) conducted until now, demonstrated that the correction, by thyroidectomy, of eSCH due to nodular goiter significantly increased body weight gain and low-density lipoprotein (LDL) cholesterol levels, with the preoperative serum TSH value being the main predictive factor. Here, we retrospectively assessed changes in body weight, BMI, and serum lipids following the correction of eSCH due to multinodular goiter by another therapeutic modality, radioactive iodine $\left({ }^{131} \mathrm{I}\right)$. In the absence of a euthyroid control group treated by ${ }^{131} \mathrm{I}$, we compared patients with grade $1 \mathrm{eSCH}$ and grade $2 \mathrm{eSCH}$ to investigate whether pretreatment grading of eSCH may predict modifications of metabolic parameters after restoration of thyroid function.

\section{Materials and Methods}

\section{Data Collection}

All consecutive files of patients treated with ${ }^{131}$ I for eSCH due to nodular goiter, identified via the Nuclear Medicine Department prospective database, between January 2008 and July 2018 and who underwent a medical follow-up in our center were retrospectively reviewed. The total study period spanned from 12 months before to 36 months after ${ }^{131} \mathrm{I}$ treatment and for the purpose of analysis was divided to 5 time points defined as 12 months before ${ }^{131}$ I treatment, just before ${ }^{131}$ I treatment (pretreatment or baseline) and 12,24 , and 36 months after ${ }^{131}$ I treatment (posttreatment follow-up). Data were assigned to a time point if they were collected within 3 months before or after this defined time point. Data were collected using Erasme Hospital ULB Brussels Medical Viewer software. Due to its retrospective nature, informed consent to collect medical file's data was not obtained from each participant and the study protocol, design, and data collection method was approved by the Erasme Hospital Ethics Committee (Comité d'Ethique Erasme-ULB, study reference number P2018/138). All available medical consultation, hospitalization records, and laboratory reports within the defined study period were examined to collect body weight measurements (weight is routinely measured with indoor clothes on analog medical scales available in every consultation area in the hospital), serum TSH and thyroid hormone levels, fasting serum cholesterol, and fasting glycemia. $\mathrm{Pa}-$ tients' height, current or past medication, smoking habits, and medical history were also collected from the medical file.

\section{Patients and Biochemical Analysis}

Patients were eligible for inclusion in this study if they had eSCH due to nodular goiter. This was confirmed by the simultaneous presence of all 3 following criteria: TSH $<0.4 \mathrm{mUI} / \mathrm{L}$ with normal fT 4 and fT3 levels, typical appearance nodular goiter by thyroid ultrasound, and presence of autonomously functioning areas demonstrated by technetium-99m pertechnetate thyroid scan. Whenever the thyroid scan appearance was not typical of an autonomous nodular goiter, we assured that TSH receptor antibody measurement was performed and was negative in order to exclude patients with Graves' disease. To allow for a clear definition of baseline thyroid status, patients treated with an antithyroid drug were not considered for this study. The minimal duration of eSCH was estimated by reporting either the oldest biological finding of $\mathrm{SCH}$ or, if not available, according to the patient's anamnesis. Patients were included in the study if at least one body weight measurement could be found in their medical file during the defined post- ${ }^{131}$ I-treatment follow-up period. Patients diagnosed with a weight-affecting condition during the follow-up period (such as cancer, bariatric surgery, corticoid treatment, or severe chronic illnesses) or deceased during the first-year post ${ }^{131}$ I were excluded. Time to achieve euthyroidism was defined as the time to obtain the first normalized $(0.4 \leq \mathrm{TSH} \leq 4 \mathrm{mUI} / \mathrm{L}) \mathrm{TSH}$ value.

At our center, TSH was measured by electrochemiluminescence using an E801 module (Roche Diagnostics) according to the manufacturer's instructions. Fasting plasma glucose, triglycerides, and total and high-density lipoprotein cholesterol were assessed using standard laboratory methods. LDL cholesterol was calculated using Friedwald's formula.

\section{Treatment}

All patients received, on an ambulatory basis, a single activity of ${ }^{131} \mathrm{I}$ which was calculated according to the following formula, considering the 24-radioidodine uptake and thyroid size, as previously described [21]: $\mu \mathrm{Ci}$ activity $=\mathrm{R}$ activity $\mu \mathrm{Ci} \times$ thyroid size $(\mathrm{g})$ 24 h uptake (\%). R activity (required activity) varied between 80 and $220 \mu \mathrm{Ci}(2.96-8.14 \mathrm{MBq}) / \mathrm{g}$ according to thyroid size.

\section{Analysis of Clinical Data}

Patients were separated into 2 groups for the analysis: 1 group with pretreatment eSCH grade $1(0.1 \leq \mathrm{TSH} \leq 0.39 \mathrm{mU} / \mathrm{L})$ and 1 with pretreatment eSCH grade $2(\mathrm{TSH}<0.1 \mathrm{mU} / \mathrm{L})$. The absolute change (difference between the post- and pretreatment) in BMI and in serum LDL cholesterol levels at 12 months posttreatment were defined as primary outcome variables of interest. The 12 months posttreatment is a typical evaluation time point after correction of eSCH, classically used in longitudinal interventional studies, and coincided with the highest availability of posttreat- 
Fig. 1. Study flowchart of patient selection. $\mathrm{eSCH}$, endogenous subclinical hyperthyroidism; TSH, thyroid-stimulating hormone.

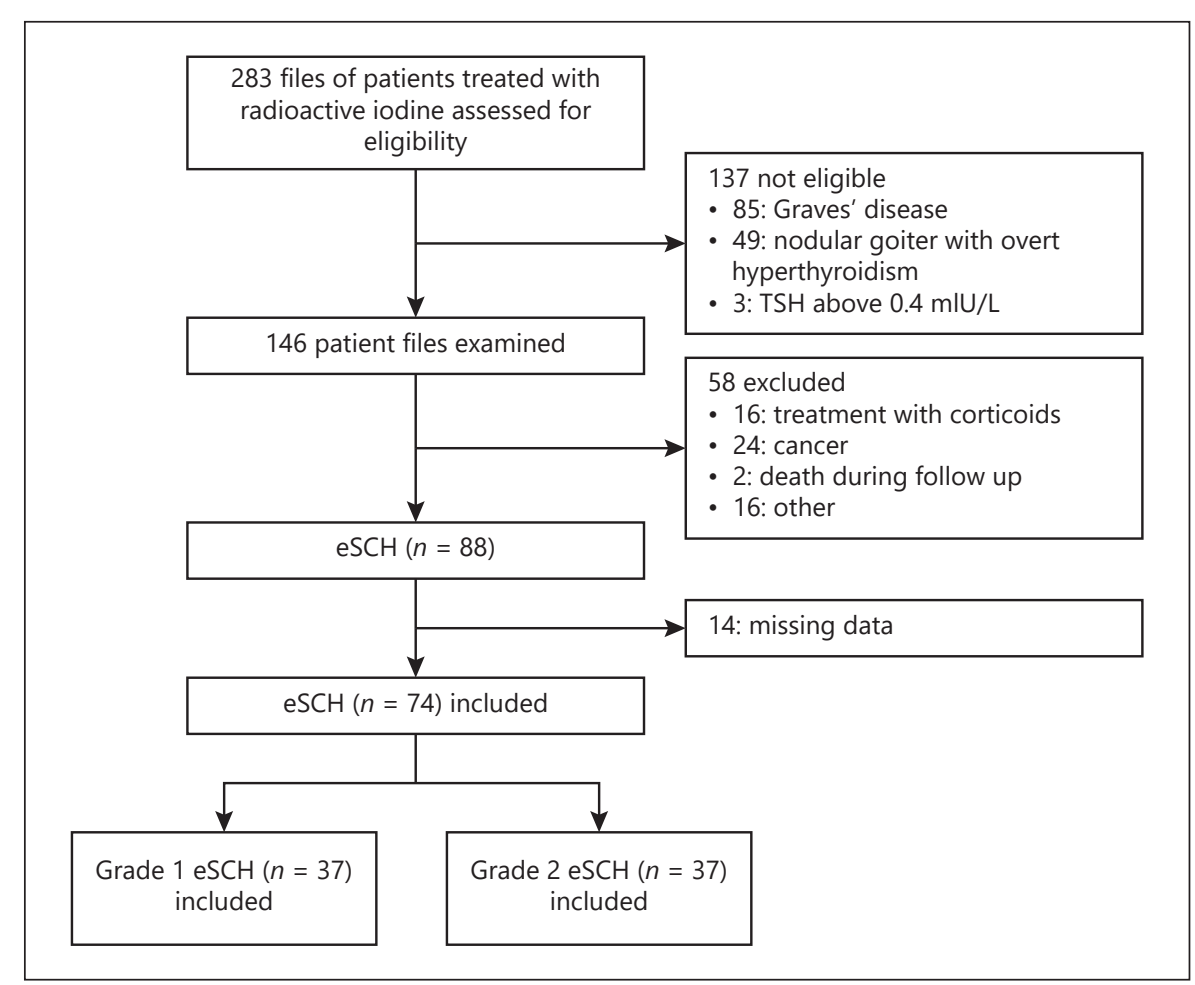

ment weight measurements in our population. It also limits the risk of influence of other factors (intercurrent illness, hospitalizations, additional medication etc.) on weight changes over time.

\section{Statistical Analysis}

Comparisons were made using the $\chi^{2}$, paired Student's $t$ test for parametric, and paired Mann-Whitney's test for nonparametric variables. We first conducted a univariate analysis of all presumed pretreatment factors associated with BMI or LDL cholesterol changes after treatment. Second, a multiple regression analysis including all variables with a correlation significance of $p<0.20 \mathrm{ob}-$ tained in the univariate analysis was performed to rule out confounding factors. Pretreatment serum TSH was tested as a continuous covariate and as a categorical variable in subgroup analyses: $0.1 \leq \mathrm{TSH} \leq 0.39 \mathrm{mU} / \mathrm{L}$ (eSCH grade 1 ) and TSH $<0.1 \mathrm{mU} / \mathrm{L}$ (eSCH grade 2). Data processing was performed using GraphPad Prism (version 6.00, La Jolla, CA, USA) and SPSS Statistics (version 25.0, IBM Corp, Armonk, NY, USA). $p<0.05$ was considered as statistically significant.

\section{Results}

All of the consecutive 146 files of patients with eSCH due to nodular goiter treated by ${ }^{131}$ I between January 2008 and July 2018 who had undergone a follow-up in our center were retrospectively reviewed (shown in Fig. 1). Fifty-eight patients with conditions likely to affect body weight during follow-up were excluded. The causes of ex- clusion were cancer $(n=24)$, corticoid treatment $(n=16)$, other $(n=16)$, or death during posttreatment follow-up $(n=2)$. Out of the 88 eligible patients, 74 had at least 1 body weight measurement in their file during the defined 12-36 months posttreatment follow-up period and were therefore included for analysis.

\section{Baseline (Pretreatment) Patients' Characteristics}

The median pretreatment TSH was $0.19 \mathrm{mU} / \mathrm{L}$ in the grade $1 \mathrm{eSCH}$ group and $0.04 \mathrm{mU} / \mathrm{L}$ in the grade $2 \mathrm{eSCH}$ group $(p<0.01)$. There were no statistically significant differences between the 2 groups regarding clinical parameters such as age, BMI, weight, sex, menopausal status ( $\geq 60$ years old or specified in medical file; 17 out of 22 female patients in grade 1 eSCH group vs. 23 out of 25 female patients in grade 2 eSCH group, $p=0.442$ ), smoking status, diabetes, duration of eSCH, statin treatment, fasting serum cholesterol and glycemia (shown in Table 1).

\section{Posttreatment Comparison}

The mean total posttreatment follow-up (29.1 \pm 1.4 vs. $30.1 \pm 1.6$ months, $p=0.297)$, the time to achieve euthyroidism (median 84 vs. 114 days, $p=0.286$, shown in Fig. 2a), and posttreatment TSH levels measured at 12, 24, and 36 months did not significantly differ between the 
Table 1. Baseline (pretreatment) characteristics of the patients

\begin{tabular}{|c|c|c|c|c|}
\hline Characteristics & $\begin{array}{l}\text { Total } \\
(n=74)\end{array}$ & $\begin{array}{l}\text { Grade } 1 \text { eSCH } \\
(n=37)\end{array}$ & $\begin{array}{l}\text { Grade } 2 \text { eSCH } \\
(n=37)\end{array}$ & $p$ value \\
\hline \multicolumn{5}{|l|}{ TSH levels, mU/L } \\
\hline Median & 0.10 & $0.19(0.13-0.27)$ & $0.04(0.01-0.07)$ & $<0.001$ \\
\hline Thyroid volume, $\mathrm{mL}$ & $41.9 \pm 4.5(n=62)$ & $47 \pm 7.9(n=32)$ & $36.4 \pm 3.6(n=30)$ & 0.713 \\
\hline FT4, pmol/L & $16.7 \pm 0.30$ & $16.22 \pm 0.45$ & $17.18 \pm 0.39$ & 0.116 \\
\hline Positive anti-TPO and/or anti-TG, $n$ & $9(n=47)$ & $3(n=24)$ & $6(n=23)$ & 0.286 \\
\hline Smoking, \% & $15.9(n=69)$ & $14.7(n=34)$ & $17.1(n=35)$ & 0.782 \\
\hline Statin use, \% & 43.2 & 45.9 & 40.5 & 0.815 \\
\hline Diabetes, \% & 31.1 & 35.1 & 27.0 & 0.616 \\
\hline Insulin use, \% & 13.5 & 16.2 & 10.8 & 0.735 \\
\hline Lipids (mg/dL) & $(n=50)$ & $(n=26)$ & $(n=24)$ & \\
\hline Fasting glycemia, mmol/L & $5.32 \pm 0.1(n=35)$ & $5.24 \pm 0.14(n=17)$ & $5.38 \pm 0.13(n=18)$ & 0.713 \\
\hline
\end{tabular}

grade 1 and grade 2 eSCH groups. At 12 months, only 6 patients ( 2 in grade 1 and 4 in grade $2 \mathrm{eSCH}$ group) needed thyroid hormone therapy and we did not observe any case of transient overt hyperthyroidism following ${ }^{131} \mathrm{I}$ treatment.

In both groups, there was a mean increase in body weight 12 months post ${ }^{131}$ I treatment (shown in Table 2). This increase was significantly higher in the grade 2 than in the grade $1 \mathrm{eSCH}$ group $(2.90 \pm 0.73$ vs. $0.65 \pm 0.39 \mathrm{~kg}$, $p=0.039)$ and was significantly different from baseline only in the grade 2 group $(p<0.01)$. Similarly, the mean increase in BMI at 12 months posttreatment was significantly higher in the grade 2 than in the grade $1 \mathrm{eSCH}$ group $\left(1.07 \pm 0.27\right.$ vs. $\left.0.26 \pm 0.15 \mathrm{~kg} / \mathrm{m}^{2}, p=0.023\right)$ and was significantly different from baseline only in the grade 2 group ( $p<0.01)$. Most of the weight gain in both groups was already observed within the first 12 months posttreatment, with no further significant differences between the 12- and 24- or 36-month-posttreatment follow-up time points (shown in Fig. 2b).

At 12 months posttreatment, serum cholesterol was available for a total of 47 patients. However, compari-

Correction of Subclinical

Hyperthyroidism Induces Weight Gain sons with baseline (meaning that both baseline and 12-month posttreatment values were available for the same patient) were possible for 38 patients in total (19 in grade 1 and 19 in grade $2 \mathrm{eSCH}$ group) (shown in Table 2). Significant increases compared to baseline were observed in the levels of total cholesterol, LDL, and triglycerides only in the grade $2 \mathrm{eSCH}$ group $(16.7 \pm 4.5$ $\mathrm{mg} / \mathrm{dL} p<0.01,14.3 \pm 4.1 \mathrm{mg} / \mathrm{dL} p<0.01,25.2 \pm 9.4 \mathrm{mg} /$ $\mathrm{dL} p=0.01$, respectively). Interestingly, although overall comparison between groups at 12 months did not show differences in lipid levels, when the nonstatin-treated population (10 patients in grade 1 and 12 in grade 2 group) was analyzed, total and LDL cholesterol significantly differed between the grade 1 and grade $2 \mathrm{eSCH}$ groups (shown in Table 2).

No further significant changes in LDL cholesterol between the 12- and 24- or 36-month-posttreatment follow-up time points were observed in either the grade 1 or grade 2 groups (data not shown). At 12 months posttreatment, fasting glycemia was available for 26 patients and no significant changes were observed in the 2 groups (shown in Table 2). 
Fig. 2. Posttreatment evolution of thyroid function and BMI in patients with grade 1 and $2 \mathrm{eSCH}$ : population with grade 1 and 2 eSCH after treatment (a) and changes in BMI during follow-up in patients with grade 1 and 2 eSCH (b). Baseline: pretreatment time point. The number of patients with available BMI data in grade $1 \mathrm{eSCH}$ was $n=23$ at $-12, n=34$ at baseline, $n=32$ at $12, n=27$ at $24, n=17$ at 36 months of follow-up and in grade 2 eSCH group, $n=$ 19 at $-12, n=32$ at baseline, $n=30$ at 12 , $n=22$ at $24, n=23$ at 36 months of follow-up, respectively. Data are reported as means \pm SEMs. ${ }^{*} p<0.05$ for comparison between patients with grade 1 and grade 2 eSCH. ${ }^{*} p<0.01$ for comparison with baseline. eSCH, endogenous subclinical hyperthyroidism.
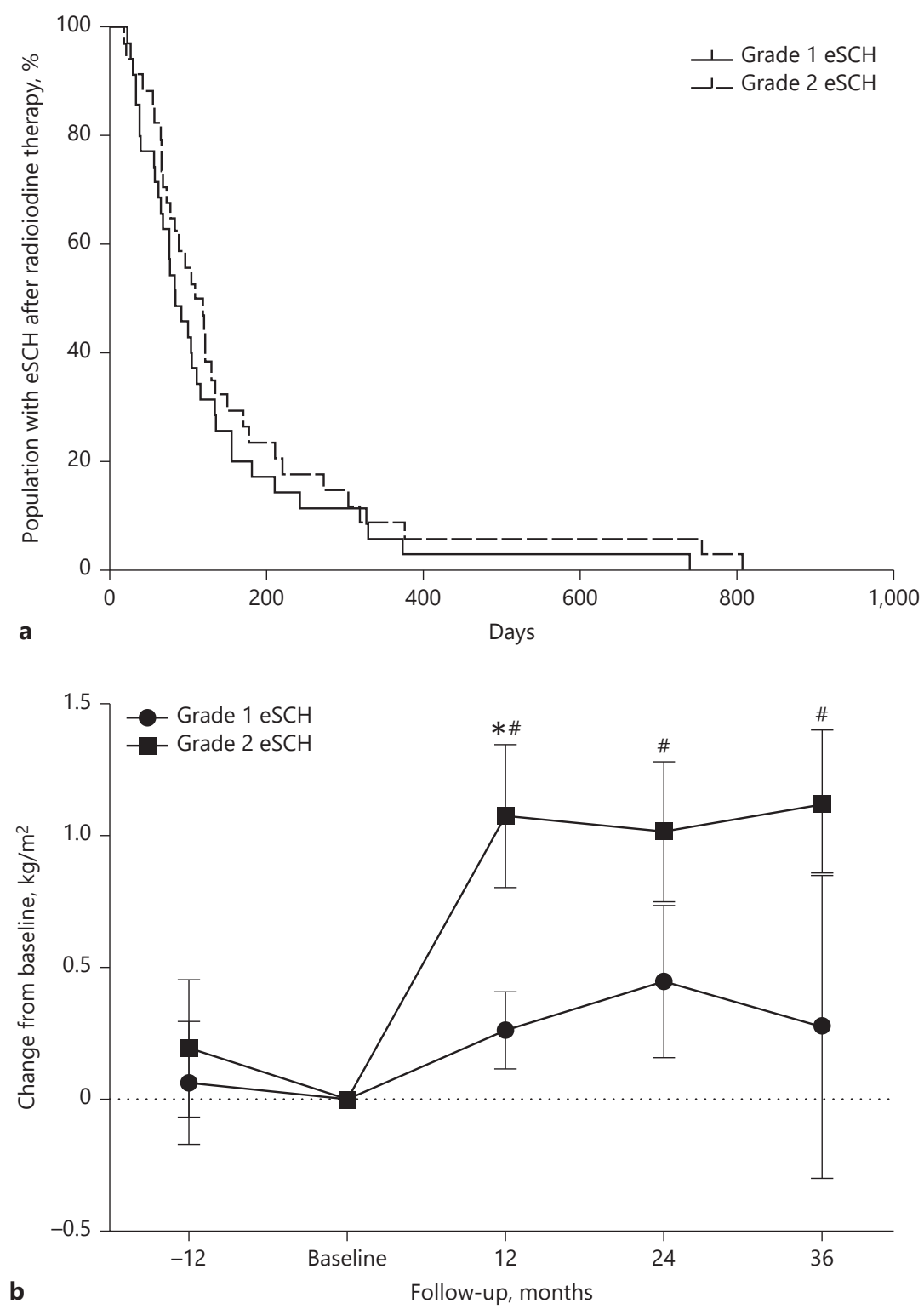

\section{Pretreatment Factors Associated with Metabolic Changes}

After adjustment for potential confounding factors, pretreatment TSH level was found to be the only factor showing a significant (negative) association with the posttreatment BMI increase in the multivariate model ( $p=0.006$ ) (online suppl. Table 1; for all online suppl. material, see www.karger.com/doi/10.1159/000512734). No significant associations were observed between pretreatment factors and LDL cholesterol increase in the univariate model. Age, smoking, female sex, and preopera- tive fT4 and fT3 levels were not significantly associated with either posttreatment increases in BMI or LDL cholesterol (shown in online suppl. Table 1).

\section{Discussion}

In patients with eSCH clearly attributed to nodular goiter, correction of eSCH by ${ }^{131} \mathrm{I}$ induces weight gain and, to a lesser extent, serum cholesterol changes with higher increases for patients with lower baseline serum 
Table 2. Posttreatment group comparisons at 12 months

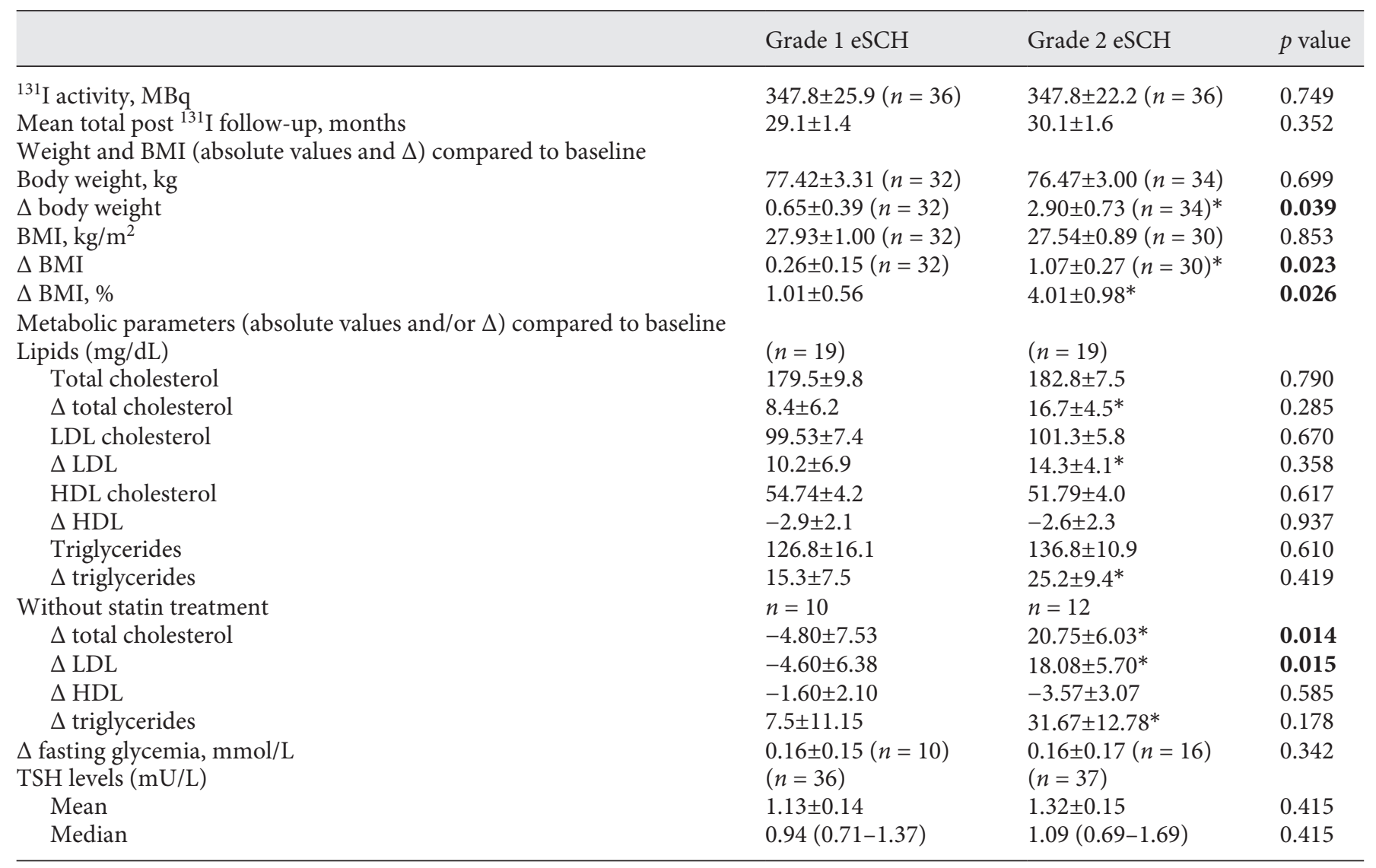

Results are mean \pm SEM or median (interquartile range). Student's $t$ test for parametric continuous variables (total cholesterol and triglycerides); Mann-Whitney test for nonparametric continuous variables (radioactive iodine dose, follow-up time, body weight, BMI, LDL cholesterol, HDL cholesterol, fasting glycemia, and TSH). When not available for all patients, $n$ represents the number of patients for whom data were available for the analysis. Significant $p$ values of the comparison between grade 1 and grade 2 eSCH are in bold. eSCH, endogenous subclinical hyperthyroidism; LDL, low-density lipoprotein; HDL, high-density lipoprotein; TSH, thyroid-stimulating hormone. ${ }^{*}$ Significant compared with baseline values $(p<0.01), \Delta$ : changes compared to baseline

$\mathrm{TSH}$. These data suggest that eSCH induces metabolic effects and confirm that some of these effects are reversible after correction of eSCH, reinforcing previous cross-sectional studies $[16,18,19]$. Moreover, we showed that the severity of subclinical hyperthyroidism - defined according to serum TSH as grade 1 or grade 2 - before treatment is the main factor to predict weight gain after treatment, suggesting that this distinction is pertinent to perform, at least in an elderly population, and can be highly relevant in clinical practice.

Large randomized clinical trials to investigate the effects of the correction of eSCH are lacking, and although undoubtedly needed, their design and conduction remain delicate and complicated in the context of official recommendations, suggesting the correction of persistent
eSCH, particularly grade 2 , at least in older patients or patients with risk factors $[5,6]$. As a result, our hereby proposal to compare the longitudinal evolution of each patient before and after correction of $\mathrm{eSCH}$ in order to assess treatment efficacy may represent an interesting alternative. In this case, one should assume a causal relationship between eSCH severity and posttreatment evolution, so grading of the disease is pertinent and necessary, assume that the treatment by itself has no effect on posttreatment evolution, and consider that the body weight in the healthy general population increases with age by approximately $0.2-0.5 \mathrm{~kg} /$ year [22].

In the present study, posttreatment increases in cholesterol level and BMI were observed only in grade 2 eSCH patients. Our results confirm the previous results 
by Bel Lassen et al. [20] although we observed a lower posttreatment increase in BMI. The differences in weight gain and cholesterol changes between the 2 studies might be explained by the following: (i) thyroid surgery results in a faster correction of hyperthyroidism than ${ }^{131} \mathrm{I}$ and may have a direct effect on weight regulation. Indeed, a weight gain of $1 \mathrm{~kg}$ was observed in the euthyroid control group, consistent with the difference observed in the 2 studies; (ii) the population treated by ${ }^{131}$ I was significantly older than that treated by surgery $(66.7 \pm 1.3$ vs. $51.5 \pm$ 1.4 years), therefore probably less healthy and more prone to diseases that may affect BMI; (iii) after surgery, patients are in a "compensated iatrogenic hypothyroidism" state and treatment with thyroxine is mandatory while after ${ }^{131} \mathrm{I}$, most patients will achieve "biological euthyroidism" without the need of exogenous administration of thyroid hormones; (iv) the posttreatment TSH levels were higher after thyroidectomy than those after ${ }^{131} \mathrm{I}$.

To our best knowledge, the several other intervention studies on the subject included a limited number of patients ( $N$ ranging from 12 to 29 ) with a follow-up evaluation either at 12 months after treatment or at 6 months post correction of SCH. While Heemstra et al. [17] reported no weight gain or cholesterol changes in exogenous $\mathrm{SCH}$ patients $(N=12)$, Greenlund et al. [18] reported a 3.8 -kg weight gain ( $N=22$ female) 6 months after the correction of eSCH. Boj-Carceller et al. [19] reported a $1.4-\mathrm{kg}$ weight gain only in a subgroup of patients aged above 65 years $(N=11)$.

In the present study, the TSH value was the main predictive factor for the increase in body weight. However, we did not find any association between pretreatment TSH and serum LDL cholesterol increase. There was no significant association between pretreatment $\mathrm{fT} 4$ or fT3 and metabolic outcomes, suggesting that the grade of severity of eSCH as reflected by the TSH value might better predict metabolic outcomes than $\mathrm{fT} 4$ or fT3. Indeed, it has previously been shown that changes in TSH within the studied $0.01 \leq \mathrm{TSH} \leq 4 \mathrm{mU} / \mathrm{L}$ range are associated with minor changes in $\mathrm{fT} 4$ and $\mathrm{fT} 3$ [23].

Our study has several limitations and weaknesses due to its retrospective design. We had no information on diet or exercise habitus before and after treatment, and posttreatment comparison of cholesterol values was possible for $50 \%$ of the patients. In addition, the weight measurements were not standardized (i.e., with or without shoes and brand of analog medical scale used). However, these methodological approximations occurred similarly in the 2 groups and resulting error from imprecise measure- ments can undermine statistical associations but does not introduce bias therefore should probably not account for the differences observed regarding weight gain. On the other hand, by comparison with cross-sectional studies, low TSH in our rather large patient population was clearly the result of eSCH as this diagnosis was proven by multiple TSH measurements and the presence of an autonomous nodular goiter. The comparable results obtained after the correction of eSCH by 2 different treatments ( ${ }^{131} \mathrm{I}$ and thyroidectomy) in 2 different populations strongly suggest, despite the retrospective design of both studies, a direct effect of eSCH on metabolism.

In conclusion, after correction of eSCH by ${ }^{131} \mathrm{I}$, increases in weight and LDL cholesterol levels were observed only in patients with pretreatment grade $2 \mathrm{eSCH}$, suggesting that it is pertinent to implement grading of eSCH in clinical practice. The pretreatment serum TSH, and not the treatment by itself, was the main independent factor associated with body weight changes. As posttreatment TSH values were similar in both groups, this observation is probably related to the severity of the disease before treatment, confirming that the distinction between grade 1 and grade 2 is clinically relevant at least for metabolic parameters in an elderly population.

\section{Acknowledgement}

We thank Wiley Editing Services for English language revisions.

\section{Statement of Ethics}

The current study complies with the guidelines for human studies and was conducted ethically in accordance with the World Medical Association Declaration of Helsinki.

Due to its retrospective nature, informed consent to collect medical file's data was not obtained from each participant and the study protocol, design, and data collection method was approved by the Erasme Hospital Ethics Committee (Comité d' Ethique Erasme-ULB, study reference number P2018/138).

\section{Conflict of Interest Statement}

The authors have nothing to disclose.

\section{Funding Sources}

This research did not receive any specific grant from any funding agency in the public, commercial, or not-for-profit sector. 


\section{Author Contributions}

L.R. and T.N.H.N. equally contributed to study design, data collection, data analysis, and manuscript drafting; A.K. contributed to critical data analysis and manuscript drafting; P.B.L. con- tributed to critical supervision of manuscript drafting; R.M. contributed to data collection; R.M.R. contributed to study design, statistical analysis, and critical supervision of manuscript drafting; and B.C. contributed to study conception and design, data analysis, and manuscript drafting.

\section{References}

1 Bjørndal MM, Sandmo Wilhelmsen K, Lu T, Jorde R. Prevalence and causes of undiagnosed hyperthyroidism in an adult healthy population. The Tromsø study. J Endocrinol Invest. 2008 Oct;31(10):856-60.

2 Laurberg P, Pedersen KM, Hreidarsson A, Sigfusson N, Iversen E, Knudsen PR. Iodine intake and the pattern of thyroid disorders: a comparative epidemiological study of thyroid abnormalities in the elderly in Iceland and in Jutland, Denmark. J Clin Endocrinol Metab. 1998 Mar;83(3):765-9.

3 Laurberg P, Cerqueira C, Ovesen L, Rasmussen LB, Perrild H, Andersen S, et al. Iodine intake as a determinant of thyroid disorders in populations. Best Pract Res Clin Endocrinol Metab. 2010 Feb;24(1):13-27.

4 Carlé A, Andersen SL, Boelaert K, Laurberg P. Management of endocrine disease: subclinical thyrotoxicosis: prevalence, causes and choice of therapy. Eur J Endocrinol. 2017 Jun; 176(6):R325-37.

5 Biondi B, Bartalena L, Cooper DS, Hegedüs L, Laurberg P, Kahaly GJ. The 2015 European thyroid association guidelines on diagnosis and treatment of endogenous subclinical hyperthyroidism. Eur Thyroid J. 2015 Sep;4(3): $149-63$.

6 Ross DS, Burch HB, Cooper DS, Greenlee MC, Laurberg P, Maia AL, et al. 2016 American thyroid association guidelines for diagnosis and management of hyperthyroidism and other causes of thyrotoxicosis. Thyroid. 2016 Oct;26(10):1343-421.

7 Biondi B, Cooper DS. Subclinical hyperthyroidism. N Engl J Med. 2018 Jun;378(25): 2411-9.

8 Cappola AR, Fried LP, Arnold AM, Danese MD, Kuller LH, Burke GL, et al. Thyroid status, cardiovascular risk, and mortality in older adults. JAMA. 2006 Mar;295(9):1033.
9 Blum MR, Bauer DC, Collet TH, Fink HA, Cappola AR, da Costa BR, et al. Subclinical thyroid dysfunction and fracture risk: a metaanalysis. JAMA. 2015 May;313(20):2055.

10 Aubert CE, Bauer DC, da Costa BR, Feller M, Rieben C, Simonsick EM, et al. The association between subclinical thyroid dysfunction and dementia: the Health, Aging and Body Composition (Health ABC) Study. Clin Endocrinol. 2017 Nov;87(5):617-26.

11 Santini F, Marzullo P, Rotondi M, Ceccarini G, Pagano L, Ippolito S, et al. Mechanisms in endocrinology: the crosstalk between thyroid gland and adipose tissue: signal integration in health and disease. Eur J Endocrinol. 2014 Oct;171(4):R137-52.

12 Hoogwerf BJ, Nuttall FQ. Long-term weight regulation in treated hyperthyroid and hypothyroid subjects. Am J Med. 1984 Jun;76(6): 963-70.

13 Peppa M, Betsi G, Dimitriadis G. Lipid abnormalities and cardiometabolic risk in patients with overt and subclinical thyroid disease. J Lipids. 2011;2011:575840.

14 Cavallo-Perin P, Bruno A, Boine L, Cassader $\mathrm{M}$, Lenti G, Pagano G. Insulin resistance in Graves' disease: a quantitative in vivo evaluation. Eur J Clin Invest. 1988 Dec;18(6):60713.

15 Torlinska B, Nichols L, Mohammed MA, McCabe C, Boelaert K. Patients treated for hyperthyroidism are at increased risk of becoming obese: findings from a large prospective secondary care cohort. Thyroid. 2019 Oct; 29(10):1380-9.

16 Knudsen N, Laurberg P, Rasmussen LB, Bülow I, Perrild H, Ovesen L, et al. Small differences in thyroid function may be important for body mass index and the occurrence of obesity in the population. J Clin Endocrinol Metab. 2005 Jul;90(7):4019-24.
17 Heemstra KA, Smit JW, Eustatia-Rutten CF, Heijboer AC, Frölich M, Romijn JA, et al. Glucose tolerance and lipid profile in longterm exogenous subclinical hyperthyroidism and the effects of restoration of euthyroidism, a randomised controlled trial. Clin Endocrinol. 2006 Dec;65(6):737-44.

18 Greenlund LJ, Nair KS, Brennan MD. Changes in body composition in women following treatment of overt and subclinical hyperthyroidism. Endocr Pract. 2008 Nov;14(8):9738.

19 Boj-Carceller D, Sanz-París A, Sánchez-Oriz E, García-Foncillas López R, Calmarza-Calmarza P, Blay-Cortes V, et al. Treatment of subclinical hyperthyroidism: effect on body composition. Nutr Hosp. 2015 Nov;32(5):2331-7.

20 Bel Lassen P, Kyrilli A, Lytrivi M, Ruiz Patino M, Corvilain B. Total thyroidectomy: a clue to understanding the metabolic changes induced by subclinical hyperthyroidism? Clin Endocrinol. 2017 Feb;86(2):270-7.

21 Kyrilli A, Tang BN, Huyge V, Blocklet D, Goldman S, Corvilain B, et al. Thiamazole pretreatment lowers the (131)I activity needed to cure hyperthyroidism in patients with nodular goiter. J Clin Endocrinol Metab. 2015 Jun;100(6):2261-7.

22 Kyriacou A, Kyriacou A, Makris KC, Syed AA, Perros P. Weight gain following treatment of hyperthyroidism-a forgotten tale. Clin Obes. 2019 Oct;9(5):e12328.

23 Baloch Z, Carayon P, Conte-Devolx B, Demers L, Feldt-Rasmussen U, Henry JF, et al. Laboratory medicine practice guidelines. Laboratory support for the diagnosis and monitoring of thyroid disease. Thyroid. 2003 Jan;13(1):3-126. 